\title{
Compressive Sensing and Sparse Antenna Arrays for Indoor 3-D Microwave Imaging
}

\author{
Simon Scott, John Wawrzynek \\ University of California, Berkeley \\ Berkeley, CA 94720, USA \\ Email: simonscott@berkeley.edu, johnw@eecs.berkeley.edu
}

\begin{abstract}
A new 3-D microwave imaging technique, based on compressive sensing, is proposed for use with sparse antenna arrays. It was designed to enable cost-effective 3-D imaging and tracking of people in an indoor environment. This algorithm is able to image both sparse and cluttered environments, through the use of wavelet transforms and compressive sensing techniques. The main advantage of the proposed technique is that it enables the use of much sparser antenna arrays than is possible with the traditional range-migration algorithm, reducing the cost of microwave imaging systems. Experiments show that the compressive sensing algorithm produced high quality 3-D images using antenna arrays that are 90 to $96 \%$ sparse. This reduces the cost of the antenna array by a factor of 10 to 25 , when compared to traditional dense arrays, without a loss in image resolution.

Index Terms-Microwave imaging, compressed sensing.
\end{abstract}

\section{INTRODUCTION}

This work focuses on large-scale indoor microwave imaging of people and furniture within a room. A planar antenna array transmits high-frequency microwaves, which reflect off these objects and are captured back at the antenna array. These backscatter reflections are processed to form a threedimensional (3-D) image of the people and other objects within the room. Such microwave imaging systems enable multiple applications within the smart home, such as hand gesture recognition, indoor positioning and motion detection. Industrial applications include 3-D robotic arm tracking.

However, few large-scale 3-D microwave imaging systems have been built to date due to cost concerns. A large antenna aperture is required for good image resolution, and most image reconstruction algorithms (such as the range migration algorithm, RMA [1]) require the antennas to be placed in 2-D grid array with sub-wavelength spacing. Violating this requirement typically results in grating lobes and poor image quality [2]. A large number of antennas are therefore required. It has been shown [3] that an array of at least $64 \times 64$ antennas is needed for the applications mentioned above, requiring over 4000 antennas and radio transceivers.

The cost of these systems can be reduced by designing each antenna and transceiver to be as low-cost as possible, through the use of low-power and low-quality components [3]. The approach taken here, however, is to use sparse antenna arrays to reduce the cost. These sparse arrays, also known as thinned arrays, have the same aperture, and hence provide the same imaging resolution, as a fully-populated array, but contain fewer antennas. If the existing fully-populated twodimensional array contained $N \times N$ antennas, then the pro- posed sparse array will contain $M \ll N^{2}$ antennas, randomly placed within the existing array aperture.

Since the standard RMA algorithm requires antennas to be placed on a dense regular grid [1], a different algorithm needs to be used to reconstruct the images. A novel compressive sensing (CS) image reconstruction algorithm was therefore developed for use with these sparse antenna arrays.

While 3-D microwave imaging using fully-populated planar arrays has been well researched [1] [4] [5], imaging using sparse antenna arrays has been less well investigated. Research into using sparse linear arrays and CS to capture 2-D images for radar applications [6] [7] has assumed that the scene is mostly empty except for one or two aircraft or other objects, which occupy only a few pixels in the image. Prior attempts to use CS with sparse planar antenna arrays for 3-D imaging [8] again assumed that the scene being imaged is sparse in the spatial domain. Unfortunately, this assumption that the environment is mostly sparse except for a few point reflectors cannot be made for indoor imaging applications, where the environment is cluttered with multiple large objects.

It will, however, be shown in this paper that these complex indoor scenes can be transformed into the wavelet domain where they do have sparse representations, allowing CS reconstruction to be performed. The novelty of this paper therefore lies in borrowing the wavelet transform from optical compressive imaging and using it to capture 3-D images of complex scenes using sparse antenna arrays.

\section{Overview of Compressive Sensing}

Compressive sensing allows a compressible signal $g$ to be captured and reconstructed when the average sampling rate is below the Nyquist threshold [9]. A signal is compressible if the information rate is much less than the signal bandwidth.

Let $w$ be the representation of signal $g$ (of length $N$ ) in the $\Psi$ domain. $g$ is compressible, or sparse, if there exists some domain $\Psi$ in which most of the coefficients of $w$ are zero, or can be set to zero without perceived loss in signal quality.

The measurement of signal $g$ is performed by correlating it with a set of measurement vectors $\left\{\Phi_{j}\right\}_{j=1}^{M}$. Each correlation gives a single measurement $m_{j}$. If the measurements are noisy with a standard deviation of $\sigma$, the original signal can be reconstructed via the optimization problem:

$$
\min _{g^{\prime}}\left\|\Psi^{*} g^{\prime}\right\|_{l 1} \text { subject to }\left\|m-\Phi g^{\prime}\right\|_{l 2} \leq \sigma
$$


where $\Psi^{*}$ is the transform into the domain where $g$ is sparse and $m$ is a vector containing all the measurements $m_{j}$.

It has been shown [10] that if the mutual coherency between $\Phi$ and $\Psi^{*}$ is small, the optimization problem will converge, with high probability, to correct solution $g$ when only a small number of measurements $M<N$ are made.

\section{Compressive Sensing FOR Microwave IMAgIng}

The proposed compressive-sensing algorithm for microwave imaging attempts to recover a 3-D image of an indoor environment from discrete radio frequency samples, collected using a sparse planar antenna array. Since it is based on the general algorithm described in Section II, the same variables will be used. The variables, in this case, represent the following:

- $g$ is the 3D image to be recovered.

- Vector $m$ contains the complex voltage samples recorded at each antenna at multiple radio frequencies.

- $\Phi$ describes how the reflected radio waves are sampled.

- $\Psi^{*}$ is the sparsifying transform.

\section{A. Sampling the Scene}

The scene must first be illuminated by a microwave signal before it can be sensed. One antenna will therefore transmit a continuous wave $(\mathrm{CW})$. This wave reflects off objects in the scene and the reflections are received by other antennas in the array. The magnitude and phase of the reflected wave is recorded at each antenna as a complex voltage and stored in vector $m$. Once all receiving antennas have been sampled, the transmitting antenna transmits the next frequency $\omega_{i}$ in a set of frequencies $\left\{\omega_{i}\right\}_{i=1}^{N_{f}}$. If there is more than one transmitting antenna, the process is repeated for each transmitter.

Standard 3-D microwave imaging algorithms, including the RMA algorithm, assume that the antennas are arranged in a fully-populated, rectangular grid with regular sub-wavelength spacing and that the frequency steps $w_{i}$ are equally spaced [4]. The CS algorithm makes the same assumption, with the exception that only a fraction of the possible antenna locations are actually populated; and only a fraction of the frequency steps are actually transmitted and sampled, as illustrated in Fig. 1. To ensure that the CS sampling function is incoherent to the sparsifying function, the antenna locations and frequency steps are chosen randomly. The backscattered wave is therefore randomly undersampled in both space and frequency.

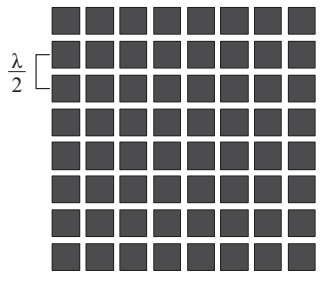

(a)

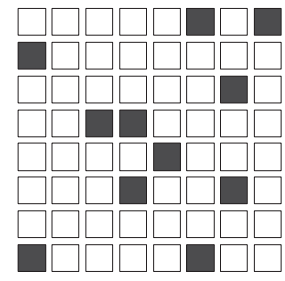

(b)
Fig. 1. (a) Fully-populated antenna array (b) Sparse array with randomlyplaced antennas, where the black squares indicate actual antenna locations

The antenna array and scene geometry is shown in Fig. 2. The antenna array lies in the $x y$-plane at $z=Z_{0}$, and contains at least one transmitting antenna and any number of receiving antennas. The reflectivity of point $(x, y, z)$ in the scene is given by function $f(x, y, z)$. The distance from antenna at location $\left(x_{a}, y_{a}\right)$ to point $\left(x_{s}, y_{s}, z_{s}\right)$ in the scene is given by:

$$
d\left(x_{a}, y_{a}, x_{s}, y_{s}, z_{s}\right)=\sqrt{\left(x_{a}-x_{s}\right)^{2}+\left(y_{a}-y_{s}\right)^{2}+\left(Z_{0}-z_{s}\right)^{2}} .
$$

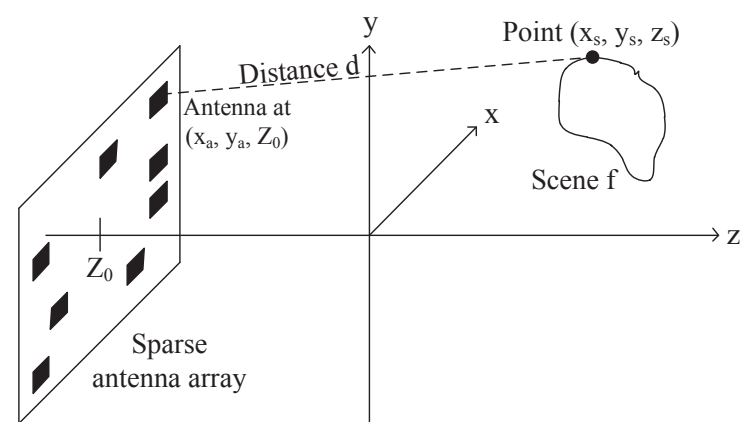

Fig. 2. The geometry of the sparse antenna array and the scene being imaged

The sampling matrix, $\Phi$, describes the relationship between the scene being imaged and the backscatter radio-frequency (RF) samples. Assume that the scene consists of a single point reflector at position $\left(x_{s}, y_{s}, z_{s}\right)$ with reflectivity $f\left(x_{s}, y_{s}, z_{s}\right)$. The reflected RF signal $m$ measured at antenna at location $\left(x_{r}, y_{r}, Z_{0}\right)$, when antenna at location $\left(x_{t}, y_{t}, Z_{0}\right)$ transmits at frequency $\omega_{i}$, is then given by:

$$
\begin{aligned}
m_{\text {point }} & \left(x_{r}, y_{r}, x_{t}, y_{t}, \omega_{i}\right)= \\
& \frac{f\left(x_{s}, y_{s}, z_{s}\right)}{d\left(x_{t}, y_{t}, x_{s}, y_{s}, z_{s}\right) d\left(x_{r}, y_{r}, x_{s}, y_{s}, z_{s}\right) k_{i}} \\
& \times e^{-j k_{i}\left(d\left(x_{t}, y_{t}, x_{s}, y_{s}, z_{s}\right)+d\left(x_{r}, y_{r}, x_{s}, y_{s}, z_{s}\right)\right)},
\end{aligned}
$$

Since $m$ represents the voltage, and hence electric field strength, at each receive antenna, the denominator term in (3) takes into account the attenuation in received field strength with distance from point reflector. The exponential term gives the round-trip phase delay and wavenumber $k_{i}=\omega_{i} / c$, where $c$ is the speed of light. Constants have been omitted.

By regarding any complex scene $f$ as a collection of point reflectors, the signal received for any arbitrary scene can be found by integrating over the scene:

$$
\begin{aligned}
& m\left(x_{r}, y_{r}, x_{t}, y_{t}, \omega_{i}\right)=\iiint_{\text {scene }} \\
& \quad \frac{f(x, y, z)}{d\left(x_{t}, y_{t}, x, y, z\right) d\left(x_{r}, y_{r}, x, y, z\right) k_{i}} \\
& \quad \times e^{-j k_{i}\left(d\left(x_{t}, y_{t}, x, y, z\right)+d\left(x_{r}, y_{r}, x, y, z\right)\right)} d x d y d z
\end{aligned}
$$

If the 3D scene to be imaged is discretized into voxels, the integrals in (4) can be replaced with summations and the entire expression can be written as a matrix-vector multiplication:

$$
m=\Phi f
$$


where the discretized scene $f$, a 3-D matrix, is vectorized for purposes of this calculation. Likewise, $m$ is a list of all the backscatter measurements taken at the different antennas and frequencies. Measurement matrix $\Phi$ contains a row for each measurement at each antenna and frequency, and a column for each point in the scene. Element $\Phi_{a, b}$ at location $(a, b)$ in matrix $\Phi$ is given by:

$$
\Phi_{a, b}=\frac{e^{-j k_{a}\left(d_{t}(a, b)+d_{r}(a, b)\right)}}{d_{t}(a, b) d_{r}(a, b) k_{a}},
$$

where $d_{t}(a, b)$ is the distance from the transmitting antenna used in measurement $a$ to point $b$ in the scene, $d_{r}(a, b)$ is the distance back to the receiving antenna and $k_{a}$ represents the microwave frequency used for measurement $a$.

These equations are for the general MIMO case, where any antenna may be arbitrarily designated a transmitter or a receiver. Simplifications can be made for single transmitter imaging systems by setting $x_{t}$ and $y_{t}$ constant; and for colocated systems (where the receiving antenna is placed immediately adjacent to the transmitting antenna) by replacing variables $x_{t}$ and $y_{t}$ with $x_{r}$ and $y_{r}$.

\section{B. Sparsifying the Image}

Although the scene may contain many objects, and hence not be sparse in the spatial domain, the surface of each object is typically made from a single material. Each object therefore appears as a solid surface with uniform intensity when imaged in the microwave spectrum. Such piecewise-constant images of real-world objects are known to be sparse in the wavelet domain [11]. The discrete wavelet transform is therefore used as the sparsifying transform, $\Psi^{*}$.

The CS algorithm for microwave imaging can be summarized as solving the following optimization problem

$$
\min _{f^{\prime}} \| \text { wavelet }\left\{f^{\prime}\right\} \|_{l 1} \text { subject to }\left\|m-\Phi f^{\prime}\right\|_{l 2} \leq \sigma \text {, }
$$

where $f^{\prime}$ is the estimate of the vectorized 3-D scene, $m$ contains the backscatter measurements, $\Phi$ is given in (6) and $\sigma$ is the standard deviation of the RF noise at the receiver. Since each row of $\Phi$ represents a randomly selected antenna or frequency, it can be shown that $\Phi$ is incoherent to the wavelet transform $\Psi^{*}[10]$, ensuring stable image recovery.

Besides being able to handle sparse antenna arrays much more efficiently and accurately than the range migration algorithm, the compressive algorithm also has the advantage of being able to handle multistatic systems where multiple transmitters are spaced multiple wavelengths apart.

\section{EXPERIMENTAL SETUP}

\section{A. Antenna Array}

A sparse antenna array was built to evaluate the CS image reconstruction algorithm. Since the exact number and placement of antennas required to image a given scene was uncertain, an XY-table was used to mechanically move a single transmit antenna and a single receive antenna to each of the random antenna locations. In this way, a large sparse antenna array was emulated using just two physical antennas. This emulation was possible because the scene remain fixed throughout the imaging process.

The XY-table and two Vivaldi antennas used to emulate the sparse array are shown in Fig. 3. It must be emphasized that the XY-table was used merely for evaluation purposes; the final system would use the same image reconstruction algorithm but with a fixed sparse array of a few hundred randomly placed antennas.

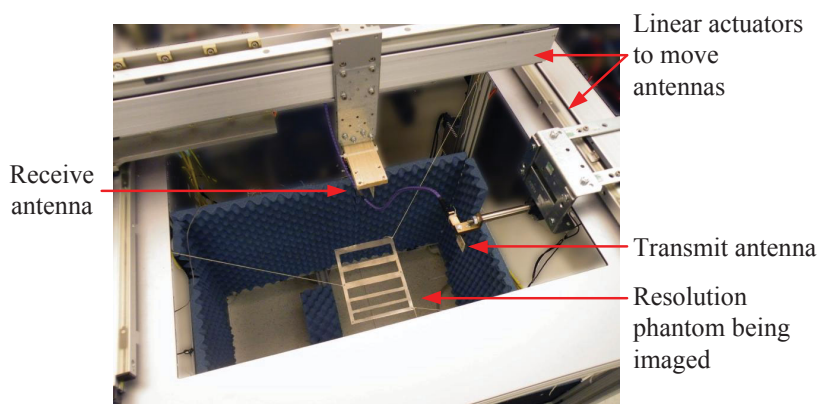

Fig. 3. The antenna array emulator.

A simple $\mathrm{CW}$ transmitter and direct conversion receiver, as shown in Fig. 4, were used to measure the reflected RF signal at each antenna location. The transceiver operates over a $3 \mathrm{GHz}$ bandwidth, from 17 to $20 \mathrm{GHz}$, with the transmit power controllable from -40 to $10 \mathrm{dBm}$. The RF frontend was intentionally kept simple so that the same circuit could be used at higher millimeter-wave frequencies.

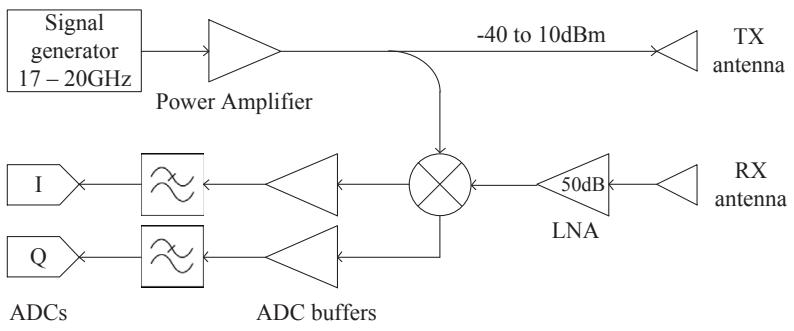

Fig. 4. The RF frontend used to transmit $\mathrm{CW}$ signals and record the backscatter.

\section{B. Performance Metrics}

To compare the quality of images produced by sparse antenna arrays to those produced by fully-populated arrays, the image signal-to-noise ratio (SNR) and image resolution were measured for different array configurations. The image SNR of the resulting 3-D images was computed using [12]

$$
S N R_{\text {image }}=\frac{\mu_{\text {object }}-\mu_{b g}}{\sigma_{b g}},
$$

where $\mu_{\text {object }}$ is the average intensity of the voxels containing the object of interest, $\mu_{b g}$ is the average intensity of the background, and $\sigma_{b g}$ is the background standard deviation. 
Image resolution was measured by imaging a phantom consisting of a set of brass strips with decreasing spacing between them, and determining the closest resolvable strips. To evaluate the feasibility of sparse arrays for hand gesture recognition, a hand phantom was created using pig skin and tissue. Both phantoms are shown in Fig. 5.

\section{Implementation of Reconstruction Algorithm}

The CS reconstruction algorithm was implemented in MATLAB, using native wavelet transforms and the SPGL1 library [13] to solve the optimization problem.

\section{IMAGING RESUltS}

The array emulator was used to evaluate a fully-populated $64 \times 64$ antenna array (4096 antennas total) with an aperture of $320 \times 320 \mathrm{~mm}$. Sparse arrays with the same aperture but consisting of 1024, 400 and 160 antennas were also emulated, representing $25 \%, 10 \%$ and $4 \%$ array densities, respectively. The following microwave imaging algorithms were compared:

- RMA: The RMA algorithm was evaluated as a baseline, with colocated transmit and receive antennas. For arrays smaller than 4096 antennas, zero filling in the missing antenna locations generally gave better results than decreasing the array aperture. This is because the random antenna placement helps to mitigate some of the grating lobes that typically result from running the RMA algorithm on sparse arrays.

- Coloc CS: The CS algorithm was evaluated on random sparse antenna arrays with colocated transmit and receive antennas. When an antenna transmits, only the antenna closest to it records the backscatter.

- MIMO CS: The CS algorithm was used with a sparse array where each receive antenna records backscatter from all other transmitters. Nine transmitters were used, evenly distributed throughout the array, for the 1024 and 400 element arrays. The sparsest array of 160 antennas consisted of 80 transmit antennas on one diagonal of the array and 80 receive antennas on the other.

The phantoms in Fig. 5 were imaged using both RMA and CS algorithms. While true 3-D images were captured, 2-D projections of these images are shown. These phantoms were placed at a distance of $0.5 \mathrm{~m}$ from the antenna array. It is clear that the CS algorithm is able to generate acceptable images of these phantoms with 400 antennas or less, while the RMA algorithm requires a dense $64 \times 64$ array of 4096 antennas.

Fig. 6 shows the image resolution that can be obtained by each of these imaging algorithms and array sizes, when the scene is $0.5 \mathrm{~m}$ from the array. The MIMO CS algorithm produced images with a $12.5 \mathrm{~mm}$ resolution with as few as 160 antennas. The Coloc CS achieved $15 \mathrm{~mm}$ resolution with 1024 antennas and $25 \mathrm{~mm}$ resolution with 400 antennas. The standard RMA algorithm produced similar resolution images, but required at least 1024 antennas. The proposed CS algorithm therefore produced higher resolution images with $6 \times$ fewer antennas than the standard RMA algorithm.

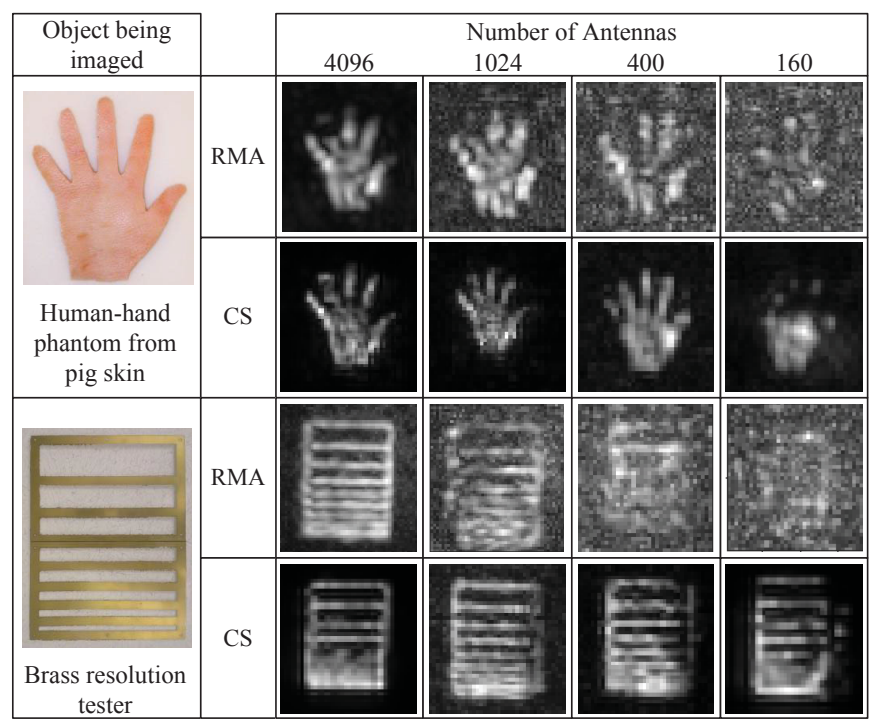

Fig. 5. Comparison of 2-D projections of 3-D images obtained using the RMA algorithm and the proposed CS algorithm for various numbers of antennas. The human hand phantom is life-size, while the brass resolution tester is $200 \mathrm{~mm}$ in length.

Of interest is the 4096 antenna array, representing a fully populated array, which allows traditional microwave imaging to be used without grating lobe issues. Therefore, in this case, the exact solution determined by the RMA algorithm produced slightly higher resolution images than the approximate solution found by the CS approach.

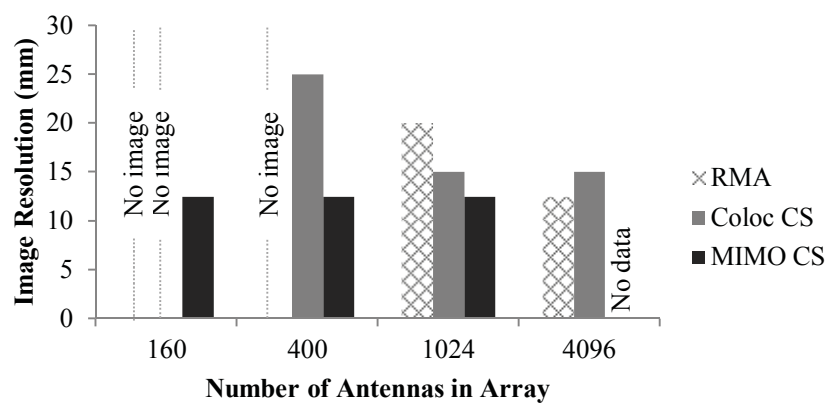

Fig. 6. Image resolution achieved by each algorithm for different array sizes

Since the RMA algorithm is known to work well at low transmit power levels [3], the CS algorithm was evaluated over a range of transmit powers. Fig. 7 shows that while the RMA algorithm running on a dense array produced good images with a transmit power as low as $-25 \mathrm{dBm}$, two of the three sparse array CS implementations were only able to operate down to $-20 \mathrm{dBm}$. This is because the sparse array contains fewer antenna elements than the dense array, and hence has lower gain [14]. Even though the minimum power required for each radio transceiver increases by a factor of $3.2(5 \mathrm{~dB})$ for the CS case, the number of transceivers decreases by at least a factor of 4, resulting in a net decrease in power consumption.

Interestingly, at higher powers, the CS algorithm produces images with a higher image SNR than the RMA algorithm, 
even though fewer antennas were used. This is because at higher transmit powers, the image SNR is limited by the image recovery algorithm, and not the received signal SNR. Hence, the decreased array gain has no effect.

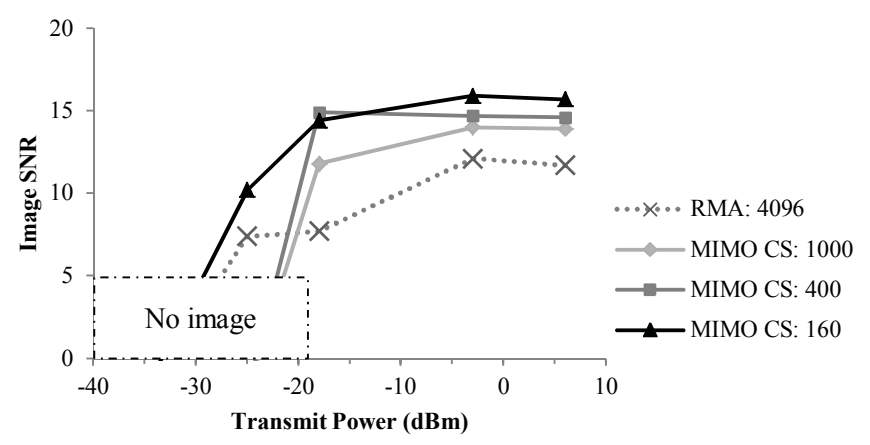

Fig. 7. The effect of transmit power on image SNR

\section{DISCUSSION}

While the proposed CS algorithm has been shown to reduce the required number of antennas, it does have two drawbacks over the traditional range migration algorithm: computational cost and tuning parameter sensitivity. On average, a MATLAB implementation of the CS algorithm was $370 \times$ slower than a MATLAB RMA algorithm, due to the computational complexity of the iterative $l_{1}$-norm minimization solver and the size of the sampling matrix $\Phi$. Other $l_{1}$-norm minimization libraries, such as NESTA [15], were investigated, but showed no performance improvement.

In practice, the CS algorithm would most likely be used in a real-time video imaging system. Therefore, the solver could be seeded with the previous video frame, provided the changes between frames are minimal, reducing computation time.

The quality of the produced images was also found to be sensitive to the noise parameter $\sigma$. While $\sigma$ was manually tuned for the results presented here, an iterative tuning algorithm can be used for real-time online applications:

- Start by setting $\sigma$ to its largest possible value, i.e. $\sigma=$ $\|m\|_{l 2}$, where $m$ is the measurement vector.

- Decrease $\sigma$ on each subsequent iteration frame until $\sigma$ is made too small, usually indicated by the optimization solver suddenly requiring a large number of iterations to converge on a solution. At this point, $\sigma$ should be increased slightly.

- Since SNR does not change much between subsequent video frames, only small changes should be required to $\sigma$ from one frame to the next.

An alternative would be to use a homotopy algorithm for CS, such as [16] [17], where each recovered video frame can be both used to seed recovery of the next frame and iteratively determine the optimal value for $\sigma$. While it would have been advantageous to evaluate the optimizations discussed here, it was not possible due to the many hours that the motorized XY-table takes to gather the data for a single frame.

\section{CONCLUSION}

Although the RMA imaging algorithm is able to produce images from an antenna array as sparse as $25 \%$, the new CS algorithm was shown to work with random sparse arrays with just $4 \%$ density. Furthermore, the CS algorithm produces higher SNR images than the RMA algorithm at all but the lowest power levels. While the reduction in the number of antennas afforded by the CS algorithm does translate into significant component cost and power consumption savings for microwave imaging systems, there may be an increased computational cost that needs to be balanced.

\section{ACKNOWLEDGMENT}

This work was supported by both the Berkeley Wireless Research Center and TerraSwarm, one of six centers of STARnet, a Semiconductor Research Corporation program sponsored by MARCO and DARPA.

\section{REFERENCES}

[1] J. Lopez-Sanchez and J. Fortuny-Guasch, "3-D radar imaging using range migration techniques," IEEE Trans. Antennas Propag., vol. 48, no. 5, pp. 728-737, May 2000.

[2] W. A. van Cappellen, S. J. Wijnholds, and J. D. Bregman, "Sparse antenna array configurations in large aperture synthesis radio telescopes," in 2006 European Radar Conference, Sept 2006, pp. 76-79.

[3] S. Scott and J. Wawrzynek, "Energy efficient antenna arrays for indoor 3-D microwave imaging," IEEE Trans. Microw. Theory Techn., vol. 64, no. 12, pp. 4801-4811, Dec 2016.

[4] D. Sheen, D. McMakin, and T. Hall, "Three-dimensional millimeterwave imaging for concealed weapon detection," IEEE Trans. Microw. Theory Techn., vol. 49, no. 9, pp. 1581-1592, Sep 2001.

[5] X. Zhuge and A. Yarovoy, "Three-dimensional near-field MIMO array imaging using range migration techniques," IEEE Trans. Image Process., vol. 21, no. 6, pp. 3026-3033, June 2012.

[6] Q. Huang, L. Qu, B. Wu, and G. Fang, "UWB through-wall imaging based on compressive sensing," IEEE Trans. Geosci. Remote Sens., vol. 48, no. 3, pp. 1408-1415, March 2010.

[7] M. Rossi, A. M. Haimovich, and Y. C. Eldar, "Spatial compressive sensing for MIMO radar," IEEE Trans. Signal Process., vol. 62, no. 2, pp. 419-430, Jan 2014.

[8] Y. Fang, B. Wang, and C. Sun, "Three-dimensional near-field microwave imaging approach based on compressed sensing," in 2015 Int Symp on Antennas and Propagation (ISAP), Nov 2015, pp. 1-4.

[9] E. J. Candes, J. Romberg, and T. Tao, "Robust uncertainty principles: exact signal reconstruction from highly incomplete frequency information," IEEE Trans. Inf. Theory, vol. 52, no. 2, pp. 489-509, Feb 2006.

[10] E. J. Candes, J. K. Romberg, and T. Tao, "Stable signal recovery from incomplete and inaccurate measurements," Communications on Pure and Applied Mathematics, vol. 59, no. 8, pp. 1207-1223, 2006.

[11] M. Lustig, D. Donoho, and J. M. Pauly, "Sparse MRI: The application of compressed sensing for rapid MR imaging," Magnetic Resonance in Medicine, vol. 58, no. 6, pp. 1182-1195, 2007.

[12] J. L. Prince and J. M. Links, Medical Imaging Signals and Systems, 1st ed. Prentice Hall, 2005, pg. 165.

[13] E. van den Berg and M. P. Friedlander, "Probing the Pareto frontier for basis pursuit solutions," SIAM Journal on Scientific Computing, vol. 31, no. 2, pp. 890-912, 2008.

[14] A. Maffett, "Array factors with nonuniform spacing parameter," IEEE Trans. Antennas Propag., vol. 10, no. 2, pp. 131-136, March 1962.

[15] S. Becker, J. Bobin, and E. J. Candès, "NESTA: A fast and accurate first-order method for sparse recovery," SIAM J. Img. Sci., vol. 4, no. 1, pp. 1-39, Jan. 2011.

[16] L. Zhang, T. Yang, R. Jin, and Z.-H. Zhou, "A simple homotopy algorithm for compressive sensing," in Proc. 18th Int. Conf. Artificial Intelligence and Statistics (AISTATS), 2015, pp. 1116-1124.

[17] P. Garrigues and L. E. Ghaoui, "An homotopy algorithm for the lasso with online observations," in Advances in Neural Information Processing Systems 21. Curran Associates, Inc., 2009, pp. 489-496. 